\title{
SPATIAL COVARIANCE IN PLANT COMMUNITIES: INTEGRATING ORDINATION, GEOSTATISTICS, AND VARIANCE TESTING
}

\author{
Helene H. WAGNeR ${ }^{1}$ \\ Department of Biology, Colorado State University, Fort Collins, Colorado 80523 USA, and \\ WSL, Swiss Federal Institute for Forest, Snow, and Landscape Research, 8903 Birmensdorf, Switzerland
}

\begin{abstract}
Spatial structure in plant communities occurs in the forms of (1) singlespecies aggregation and dispersion patterns, (2) distance-dependent interactions between species, and (3) the response to the spatial structure of environmental conditions. Different methods deal with these components of spatial variation: geostatistical analysis reveals autocorrelation in a spatial sample; the variance of species richness has been used as an indicator for interspecific interactions due to niche limitation; and ordination techniques describe multispecies responses to environmental factors. Based on the mathematical properties of presence-absence data, it is shown how variogram modeling, the testing of interspecific associations, and multiscale ordination can be integrated using the same set of distance-dependent variance-covariance matrices (variogram matrix). The variogram matrix partitions the variance of community data into spatial components at the levels of the individual species, species composition, and species richness. It can be used to factor out the effects of single-species aggregation patterns, interspecific interactions, or environmental heterogeneity. The mathematical integration of traditionally unrelated methods increases the interpretability of variograms of plant communities, provides a spatial extension and an empirical null model for the variance test of species richness, and extends multiscale ordination to nonsystematic spatial samples. Beyond the individual applications, the variogram matrix provides a framework for a mathematical unification of geostatistics, multivariate data analysis, and the analysis of variance that may enable ecologists from a broad range of fields to incorporate spatial effects into their research and to integrate analyses across different levels of biological organization.
\end{abstract}

Key words: interspecipc associations; multiscale ordination; multivariate geostatistics; nonstationarity; spatial variance; species richness; variance test; variogram matrix.

\section{INTRODUCTION}

Differences in species composition between sampling units, such as quadrats, are a primary focus of quantitative vegetation analysis. Ordination is the main method used for analyzing variation in plant communities. Indirect ordination detects intrinsic gradients in species composition, while direct gradient analysis identifies compositional gradients in vegetation as a response to measured environmental factors (De'ath 1999). Plant communities and environmental factors often are spatially structured. Direct and indirect ordination, however, are both essentially nonspatial methods

Due to an increasing awareness of the importance of space in ecology and the availability of global positioning systems (GPS), more and more data sets are spatially referenced and lend themselves to spatial analysis. If the data represent a transect or grid of contiguous quadrats, their spatial structure can be analyzed by block-size variance analysis, also known as pattern

Manuscript received 23 August 2001; revised 25 July 2002; accepted 1 September 2002. Corresponding Editor: D. W. Roberts

${ }^{1}$ Present address: WSL (see above).

E-mail: helene.wagner@wsl.ch analysis (e.g., Greig-Smith 1952, Hill 1973, Usher 1975, Dale 1999). Block-size variance techniques summarize the spatial structure of individual species and of pairs of species; they are essentially uni- or bivariate methods (Fortin 1999, Mistral et al. 2000). The respective scales of regular spatial patterns in a community, such as patches and gaps of constant size, are identified by comparing several uni- or bivariate plots of variance against block size. Alternatively, pattern analysis can be performed on the scores of an ordination axis (Galiano 1983).

A truly multivariate extension, called multiscale ordination, was presented by Noy-Meir and Anderson (1971) and further developed by Ver Hoef and GlennLewin (1989). Noy-Meir and Anderson (1971) suggested summarizing the spatial structure of a community by calculating a variance-covariance matrix for each block size. In order to facilitate interpretation, the matrices are added to form a combined covariance matrix, which is subjected to principal component analysis (PCA). The scales of spatially overlapping, statistically uncorrelated multispecies patterns are identified by partitioning the variance of each PCA axis by block size.

Increasingly, ecologists are exploring the possibilities of geostatistical analysis (e.g., Burrough 1987, Palmer 1988, Legendre and Fortin 1989, Rossi et al. 
1992, Fortin 1999, Koenig 1999). The geostatistical approach is based on distance rather than block size, which has the advantage that the quadrats need not be contiguous, nor spaced at regular intervals. The spatial structure of a dataset is usually described by an empirical variogram, which is basically a plot of the variance or difference between pairs of observations against their distance in geographic space. A variogram can be interpreted in a similar way as a plot of variance against block size derived by blocked variance techniques (Ver Hoef et al. 1993). In addition to descriptive purposes, variogram modeling can be used to interpolate point observations by kriging (Isaaks and Srivastava 1989, Cressie 1991, Haining 1997). However, like pattern analysis, geostatistical analysis has been applied mostly to single variables such as species richness or the scores of quadrats along a major ordination axis (Palmer 1988, Legendre 1993, Jonsson and Moen 1998).

Several authors have proposed ways of plotting some kind of resemblance measure (a multispecies measure of the similarity or dissimilarity of pairs of quadrats) against geographic distance. Examples are the Mantel correlogram (Sokal 1986), the method of Nekola and White (1999) for determining the rate of distance decay, or the "dissimilogram" proposed by Mistral et al. (2000). Such plots provide a very flexible description of the overall multivariate spatial structure of a community. However, generalized variograms cannot be used for interpolation, and their ecological interpretation is limited as their behavior is typically unknown.

Spatial structure in plant communities arises from a variety of factors. These factors fall into three broad groups: (1) morphological factors, such as plant size or dispersal mechanism, which influence the spatial aggregation within a population; (2) interspecific interactions within a community; and (3) the response to environmental factors, which themselves are spatially structured (Kershaw 1964, Dale 1999, Koenig 1999). Based on hierarchy theory (Allen and Starr 1982), Legendre (1993) postulated that physical processes create environmental heterogeneity at broad scales, while contagious biotic processes may cause further structuring within smaller areas of relative environmental homogeneity. This hierarchical view implies that one may assume local homogeneity within a study area even though heterogeneity exists at a larger scale. If, however, there are no independent domains of scale but the scales of physical and biotic processes overlap, we need to account for environmental heterogeneity when investigating patterns caused by biotic processes, and vice versa.

Although it is common to use more than one spatial or nonspatial method to analyze the same dataset, the techniques are usually applied individually rather than in an integrated way. On a conceptual level, this means that we investigate factors determining community structure individually, ignoring the contributions of other factors and their potential interactions. However, one cannot answer the question of why communities vary and, ultimately, why species coexist, without understanding how various factors interact and what determines their relative importance. For example, testing the variance of quadrat species richness against a null model has often been used by ecologists as a test of interspecific association (for a review, see Palmer and Van der Maarel 1995), but the difficulties of accounting for spatial autocorrelation, for the distance-dependence of species associations, and for environmental heterogeneity severely limit the capacity of this method to provide evidence for niche limitation (Palmer and van der Maarel 1995, van der Maarel et al. 1995, Wilson et al. 1995, Roxburgh and Matsuki 1999).

This paper presents a mathematical unification of geostatistical analysis, the analysis of interspecific associations, and multiscale ordination. The integration provides a framework for partitioning the variance in community data into the distance-dependent components of single-species aggregation patterns, interactions between species, and species-specific responses to environmental gradients. Starting with a mathematical model of species richness as the sum of a set of binary species variables, I reexpress the variance of species richness in terms of spatial covariance, which is conveniently summarized in the variogram matrix. I derive a standardized variogram for binary data that makes spatial covariance observed under different environmental conditions directly comparable. I present a spatial extension of the variance test of species richness that accounts for spatial autocorrelation and the distance-dependent nature of interspecific associations, and I show how multiscale ordination can be used to remove variance attributed to a larger scale trend. Understory vegetation data from the Oosting Natural Area in North Carolina serve to highlight the ecological application, and a worked example on artificial data in the Appendix illustrates the calculations.

\section{Model Structure And Methods}

\section{A mathematical model of species richness}

The following mathematical model reflects the fact that species richness is not a simple quantitative variable, but a result of the distributions of individual, interdependent species. Statistically speaking, the occurrence of a species $i$ in a single quadrat is itself a random variable $x_{i}$ with a specific probability distribution. Therefore, I start with the formal definitions of species occurrence, species composition, and species richness for a single quadrat.

Let $x_{i}$ be a binary variable that takes the value 1 if species $i$ is present in a quadrat, and 0 if it is absent. The defining parameter $p_{i}$, the mean or probability of occurrence of $i$ in the quadrat, will depend on quadrat size and shape: 


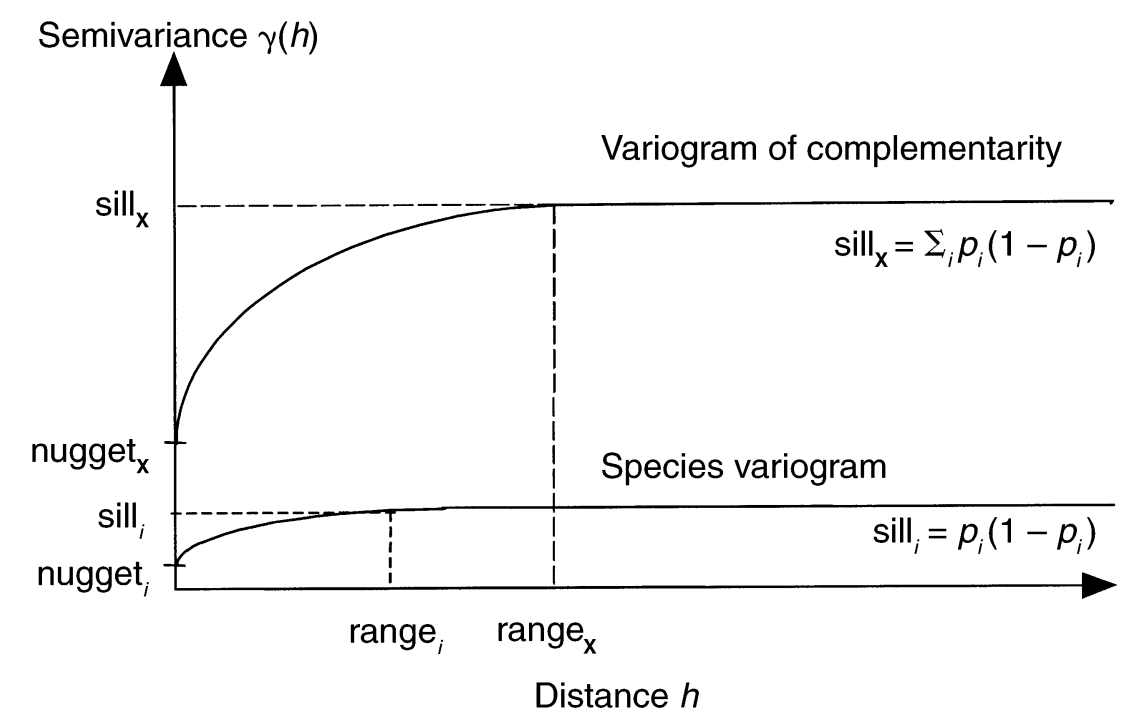

FIG. 1. Hypothetical variograms of the complementarity of species composition, $\mathbf{X}$, and of a single species $i$. The parameters sill, range, and nugget of the variogram of complementarity are related to the respective parameters of the variograms of the constituent species.

$$
x_{i}=\left\{\begin{array}{cc}
1 & p_{i} \\
0 & 1-p_{i}
\end{array}\right\} \quad i \in\{1, \ldots, s\} \quad 0 \leq p_{i} \leq 1
$$

Species composition $\mathbf{X}$ is the vector of $s$ binary variables $x_{i}$ that describe the occurrence of $s$ species in the quadrat. Quadrat species richness $S$ is the sum of the vector of species composition $\mathbf{X}$, i.e., the sum of all species variables $x_{i}$ :

$$
S=\sum \mathbf{X}=\sum_{i} x_{i}
$$

The expected value of $S$ is the sum of the expected values of the binary variables $x_{i}$, even if the variables are not independent. The variance of $S$ is the sum of the variance-covariance matrix:

$$
\begin{aligned}
\operatorname{Var}(S) & =\sum_{i=1}^{s} \operatorname{Var}\left(x_{i}\right)+2 \sum_{i=2}^{s} \sum_{j=1}^{i-1} \operatorname{Cov}\left(x_{i}, x_{j}\right) \\
& =\sum_{i=1}^{s} \sum_{j=1}^{s} \operatorname{Cov}\left(x_{i}, x_{j}\right) \quad i, j \in\{1, \ldots, s\} .
\end{aligned}
$$

If the species variables are independent, the pairwise covariances between all species $i$ and $j$ are zero and the variance of species richness $S$ is equal to the variance of species composition $\mathbf{X}$, which is the sum of the variances of the $s$ species variables $x_{i}$.

In practice, it is not possible to observe multiple independent realizations of $x_{i}$ in a single quadrat under exactly the same conditions. Hence, one cannot estimate the statistical properties of $x_{i}$ (or of $S$ ) directly from empirical data from a single quadrat. One solution is to observe one realization each of a number of random variables $x_{i a}$, with $a \in\{1, \ldots, N\}$, from a sample of $N$ quadrats, assuming the variables $x_{i a}$ to be inde- pendent and identically distributed with the probability distribution function of Eq. 1. Thus the quadrats act as replicates, and the variances and covariances in Eq. 3 are estimated from the replicated data. What happens if the quadrats are not true replicates, either because they are not independent due to spatial autocorrelation, or because they are not comparable due to environmental heterogeneity, is the primary subject of this paper.

\section{Spatial covariance}

Geostatistical methods deal with the question of how variance and covariance depend on the distance between observations (i.e., quadrats). Spatial autocorrelation, or distance dependence, is commonly modeled by fitting a variogram function to an empirical variogram (Isaaks and Srivastava 1989, Cressie 1991, Haining 1997, Burrough and McDonnell 1998). An empirical variogram is a plot of half the squared difference between two observations (the semivariance) against their distance in space, averaged for a series of distance classes. A simple variogram model is defined by the model family and the parameters sill (the average half squared difference of two independent observations), range (the maximum distance at which pairs of observations will influence each other), and nugget (the variance within the sampling unit; Fig. 1).

The most commonly used model families (spherical, exponential, and Gaussian models) assume that there is no spatial dependence for distances larger than the range. However, ecological communities may be organized in periodic spatial patterns, which result in a cyclic pattern of variance plotted against distance. This can be modeled with a hole effect model, a dampening 
sine function that is defined by the nugget effect and the range and eventually stabilizes at the sill (Legendre and Legendre 1998).

Variogram modeling is well developed for metric (ordinary kriging) and binary (probability kriging) uniand bivariate data, but no standard methods exist for the multivariate categorical data that are typical for plant community ecology. Hence, I will first develop the empirical variogram description of a set of binary variables $x_{i}$ as observed in a sample of $N$ quadrats.

An omnidirectional empirical variogram of a single variable $x_{i}$ is constructed by estimating the empirical semivariance, $\hat{\gamma}_{i}(h)$, for a range of distance classes $h$ (Isaaks and Srivastava 1989, Cressie 1991):

$$
\hat{\gamma}_{i}(h)=\frac{1}{2 n_{h}} \sum_{a, b \mid h_{a b} \approx h}\left(x_{i a}-x_{i b}\right)^{2}
$$

where $n_{h}$ is the number of pairs of quadrats $a$ and $b$ separated by approximately $h$. Sample size $n_{h}$ decreases with large distances $h$. Distances greater than half the maximum extent of the study area can only be observed for quadrats outside of the center of the study area. This introduces a bias, as the quadrats from the center do not contribute to variance estimates for larger distances. Therefore, interpretation is commonly limited to distances smaller than an arbitrary maximum distance $h_{\max }$ :

The univariate definition of a variogram can be extended to multivariate data, in which case $x_{a}$ and $x_{b}$ are not two observations of a single variable $x$, but vectors $\mathbf{X}_{a}$ and $\mathbf{X}_{b}$ of two observations of $s$ variables $x_{i}$. The empirical semivariance $\hat{\gamma}(h)$ becomes half the squared Euclidean distance between $\mathbf{X}_{a}$ and $\mathbf{X}_{b}$ and is equal to the sum of the empirical semivariances $\hat{\gamma}_{i}(h)$ of the species variables $x_{i}$ :

$$
\begin{aligned}
\hat{\gamma}(h) & =\frac{1}{2 n_{h}} \sum_{a, b \mid h_{a b} \approx h}\left\|\mathbf{X}_{a}-\mathbf{X}_{b}\right\|^{2} \\
& =\sum_{i} \frac{1}{2 n_{h}} \sum_{a, b \mid h_{a b} \approx h}\left(x_{i a}-x_{i b}\right)^{2}=\sum_{i} \hat{\gamma}_{i}(h) .
\end{aligned}
$$

In the case of binary variables, $\hat{\gamma}(h)$ equals the mean number of species that are present in only one of a pair of observations, regardless of the direction of comparison. It is a direct measure of species turnover and describes the complementarity of the species composition of two quadrats. Therefore, $\hat{\gamma}(h)$ will be referred to as the variogram of complementarity. The concept of complementarity is statistically equivalent to the distinctness or dissimilarity of species composition. As a concept, however, it captures the sense that complementary faunas or floras form parts of a whole, so that complementarity is a positive biodiversity component (Vane-Wright et al. 1991, Colwell and Coddington 1994).

In order to obtain a spatial definition of the variance of species richness, the covariances between species need to be expressed in terms of cross-variograms. An empirical cross-variogram $\hat{\gamma}_{i j}(h)$ describes the distancedependent covariance between two species, $i$ and $j$ (Isaaks and Srivastava 1989):

$$
\hat{\gamma}_{i j}(h)=\frac{1}{2 n_{h}} \sum_{a, b \mid h_{a b} \approx h}\left(x_{i a}-x_{i b}\right)\left(x_{j a}-x_{j b}\right) .
$$

Based on Eqs. 3 and 6, the variance of species richness can be expressed as spatial covariance (note that the "semivariance" in a variogram is equal to the variance of a single species occurrence, complementarity, or species richness):

$$
\begin{aligned}
\widehat{\operatorname{Var}}(S) & =\frac{1}{n} \sum_{i j} \sum_{a b} \frac{\left(x_{i a}-x_{i b}\right)\left(x_{j a}-x_{j b}\right)}{2} \\
& =\sum_{h} \frac{n_{h}}{n} \hat{\gamma}_{S}(h)=\sum_{h} \frac{n_{h}}{n} \sum_{i j} \hat{\gamma}_{i j}(h) .
\end{aligned}
$$

As the worked example in the Appendix shows, Eq. 7 provides the empirical variance of species richness $S$. In geostatistical analysis, however, only distances $>0$ are analyzed, so that a quadrat is never compared to itself. Under this condition that $a \neq b, N$ quadrats will provide $n=N(N-1) / 2$ unique pairs of quadrats $a$ and $b$, and Eq. 7 results in the unbiased estimator of the variance of species richness $S$.

Eq. 7 includes the definitions of the variance of complementarity, pairs of species, and an individual species as special cases. The condition $i=j$ leads to the variance of complementarity; for a given pair of species $i$ and $j$, Eq. 7 results in their cross-variogram; and for a single species $i=j$, it describes the variance of the variable $x_{i}$.

The basic element of spatial covariance, $\hat{\gamma}_{i j}(a, b)$, follows from Eq. 7. It is half the product of the observed differences between quadrats $a$ and $b$ for two species $i$ and $j$ :

$$
\hat{\gamma}_{i j}(a, b)=\frac{1}{2}\left(x_{i a}-x_{i b}\right)\left(x_{j a}-x_{j b}\right) .
$$

\section{The variogram matrix}

The spatial covariance can be summarized in a set of distance-dependent variance-covariance matrices $\mathbf{C}(h)$. The matrix elements $c_{i j}(h)=\hat{\gamma}_{i j}(h)$ are calculated from Eq. 6. The term variogram matrix of Myers (1997) will be used for such a set of distance-dependent variance-covariance matrices $\mathbf{C}(h)$. The variogram matrix can be interpreted in various ways (Fig. 2):

1) A plot of a diagonal element $c_{i i}(h)$ against distance $h$ is the empirical variogram of species $i$. Its expected value for independent observations in a homogeneous environment is $p_{i}\left(1-p_{i}\right)$.

2) A plot of $c_{i j}(h)$ against distance $h$ is the empirical cross-variogram of species $i$ and $j$. Its expected value for independent observations in a homo- 


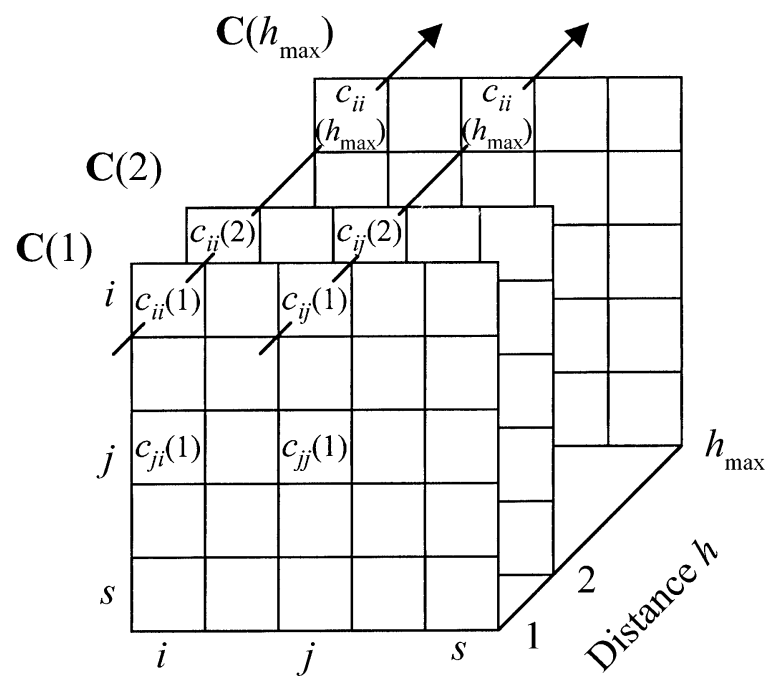

FIG. 2. Schematic representation of a variogram matrix. A variogram matrix contains a separate variance-covariance matrix $\mathbf{C}(h)$ for each distance class $h$, up to an arbitrarily defined maximum distance $h_{\max }$. Each cell of a matrix $\mathbf{C}(h)$ contains an estimate of the variance of one species $i$ [diagonal cells, $\left.c_{i i}(h)\right]$ or of the covariance of a pair of species $i$ and $j$ [off-diagonal cells, $c_{i j}(h)$ ], based on all pairs of observations that are separated by a distance of approximately $h$. The arrows indicate further geostatistical interpretations that are discussed in the text.

geneous environment and independent species is zero.

3) A plot of the sum of the diagonal of $\mathbf{C}(h), \Sigma_{i} c_{i i}(h)$, against distance $h$ is the empirical variogram of complementarity. Its expected value for independent observations in a homogeneous environment is $\Sigma_{i} p_{i}\left(1-p_{i}\right)$.

4) A plot of the sum of $\mathbf{C}(h), \Sigma_{i j} c_{i j}(h)$, against distance $h$ is the empirical variogram of quadrat species richness $S$. Its expected value for independent observations in a homogeneous environment and independent species is $\Sigma_{i} p_{i}\left(1-p_{i}\right)$.

Often, researchers want to test whether the observed autocorrelation is significantly different from random. A randomization test is constructed by randomly permuting the observations. The easiest way is to permute the couplets of $x$ and $y$ coordinates (Legendre and Legendre 1998). For each of $r$ permutations, a variogram matrix is derived, providing a reference distribution of $r$ values for each matrix element under the null hypothesis of spatially independent observations.

\section{The standardized variogram}

For binary variables such as species presence-absence data, the expected variance is a function of the mean. This can be used to make variograms from different areas with varying environmental conditions comparable by scaling the observed spatial covariance $\gamma_{i j}(a, b)$ in terms of the expected spatial covariance. The method corresponds to a pairwise relative vario- gram (Isaaks and Srivastava 1989), and results in a standardized variogram with an expected sill of 1 , independent of the mean and variance of a species.

The variance of a binary species variable $i$ with an underlying mean of $p_{i}$ is $p_{i}\left(1-p_{i}\right)$. Hence the standardized variogram $\gamma_{i}^{\prime}(h)$ of species $i$ is

$$
\gamma_{i}^{\prime}(h)=\frac{1}{2 n_{h}} \sum_{a, b \mid h_{a b} \approx h} \frac{\left(x_{i a}-x_{i b}\right)^{2}}{p_{i}\left(1-p_{i}\right)} .
$$

If the mean $p_{i a}$ of species $i$ depends on the quadrat $a$, the expected semivariance $\gamma_{i j}(a, b)$ of a pair of quadrats $a$ and $b$ is

$$
E\left\{\gamma_{i j}(a, b)\right\}=\frac{p_{i a}\left(1-p_{i b}\right)+p_{i b}\left(1-p_{i a}\right)}{2}
$$

and Eq. 9 extends to

$$
\gamma_{i}^{\prime}(h)=\frac{1}{n_{h}} \sum_{a, b \mid h_{a b} \approx h} \frac{\left(x_{i a}-x_{i b}\right)^{2}}{p_{i a}\left(1-p_{i b}\right)+p_{i b}\left(1-p_{i a}\right)} .
$$

If $p_{i}$ is estimated from the data, the unbiased variance estimator should be used for the denominator. This means that $c_{\mathrm{ii}}^{\prime}(h)$ in Eq. 9 needs to be multiplied by $(N-1) / N$, where $N$ is the total number of quadrats in the sample:

$$
\begin{aligned}
\hat{p}_{i} & =\frac{1}{N} \sum_{a} x_{i a} \\
\gamma_{i}^{\prime}(h) & =\frac{N-1}{N} \frac{1}{2 n_{h}} \sum_{a, b \mid h_{a b} \approx h} \frac{\left(x_{i a}-x_{i b}\right)^{2}}{\hat{p}_{i}\left(1-\hat{p}_{i}\right)} .
\end{aligned}
$$

The standardized variogram of complementarity, $\gamma^{\prime}(h)$, with estimated means $\hat{p}_{I}$ is

$$
\gamma^{\prime}(h)=\frac{N-1}{N} \frac{\sum_{i} \gamma_{i}(h)}{\sum_{i} \hat{p}_{i}\left(1-\hat{p}_{i}\right)} .
$$

Under the assumption of stationarity, the autocorrelation function $r_{i}(h)$ of a species $i$ can be expressed in terms of its semivariance $\gamma_{i}(h)$ and its semivariance for independent samples, $\gamma_{i}(\infty)$, i.e., its global variance or sill (Isaaks and Srivastava 1989):

$$
r_{i}(h)=\frac{\gamma_{i}(\infty)-\gamma_{i}(h)}{\gamma_{i}(\infty)}=1-\frac{\gamma_{i}(h)}{\gamma_{i}(\infty)} .
$$

Under the assumption that the underlying means $p_{i}$ or $p_{i a}$ are known or correctly estimated, the standardized variogram eliminates the effect of nonconstant means and variances. This means that the stationarity criteria are met and the standardized semivariance $\gamma_{i}^{\prime}(h)$ provides an estimator for the autocorrelation function $\hat{r}_{i}(h)$ :

$$
\hat{r}_{i}(h)=1-\gamma_{i}^{\prime}(h) .
$$

Under the same conditions, the standardized crosscovariance $\gamma_{i j}^{\prime}(h)$ of two species $i$ and $j$ is an estimator of their cross-correlation function $\hat{r}_{i j}(h)$ : 


$$
\begin{aligned}
\hat{r}_{i j}(h) & =\gamma_{i j}^{\prime}(h) \\
& =\frac{N-1}{N} \frac{1}{2 n_{h}} \sum_{a, b \mid h_{a b} \approx h} \frac{\left(x_{i a}-x_{i b}\right)\left(x_{j a}-x_{j b}\right)}{\sqrt{\hat{p}_{i}\left(1-\hat{p}_{i}\right)} \sqrt{\hat{p}_{j}\left(1-\hat{p}_{j}\right)}} .
\end{aligned}
$$

\section{Testing the spatial covariance of species richness}

The observed spatial covariance of complementarity provides an empirical null model of species independence for the spatial covariance of species richness. The two are affected by spatial aggregation in the individual species variables and species-specific response to environmental heterogeneity in exactly the same way. The only difference is in the associations or covariances between species, which again can be distance dependent. The test statistic is the ratio of the observed and the expected variance, as in the nonspatial variance test of species richness (Schluter 1984, McCulloch 1985, Palmer and Van der Maarel 1995). A variance ratio smaller than 1 is a potential indicator for niche limitation. The ratio can be derived by first calculating the empirical variograms of species richness ("observed variance") and of complementarity ("expected variance") before dividing the aggregate values for each distance class. Alternatively, the observed variance can be divided by the expected one for each pair of observations, as in a pairwise relative variogram (Isaaks and Srivastava 1989). A permutation test for the null hypothesis of independent species is constructed by independently permuting each species vector $x_{i}$.

\section{The spatial structure of ordination axes}

Multiscale ordination has been proposed for investigating the scale of multispecies patterns, such as a cyclic spatial structure of a gradient in species composition. In multiscale ordination (Noy-Meir and Anderson 1971, Ver Hoef and Glenn-Lewin 1989, Dale 1999), blocked-variance techniques are used to calculate a variance-covariance matrix $\mathbf{C}(b)$ of the species for each of a range of block sizes $b$. The matrices are then summed into an overall variance-covariance matrix $\mathbf{C}$, which is subjected to principal components analysis (PCA). For each PCA axis, the associated variance is partitioned by block size and plotted against block size.

The method can be adapted to the concept of spatial covariance and the distance-dependent variance-covariance matrices $\mathbf{C}(h)$ of a variogram matrix. A matrix $\mathbf{C}(0)$ for distance class $h=0$ is included and all matrices $\mathbf{C}(h)$ are weighted with $w_{h}=n_{h} / n$. The summed matrix $\Sigma_{h} w_{h} \mathbf{C}(h)$ is equal to the global empirical variance-covariance matrix $\mathbf{C}$ that is used in PCA. The eigenvalue $\lambda_{f}$ of PCA axis $f$ is partitioned among distance classes, $h$, by multiplying its associated eigenvector $\mathbf{u}_{f}$ with each of the matrices $\mathbf{C}(h)$ (cf. worked example in the Appendix):

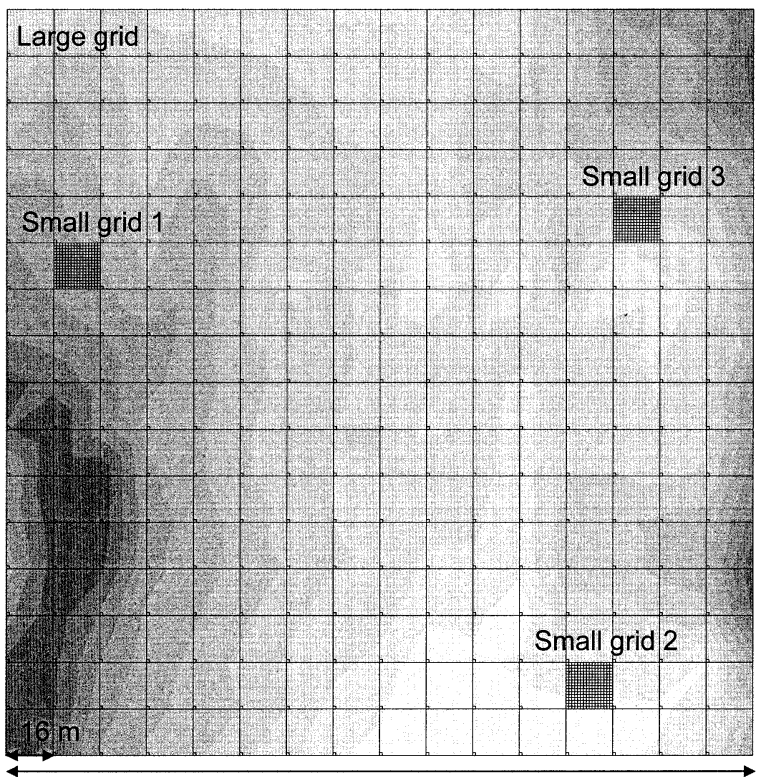

$256 \mathrm{~m}$

FIG. 3. Grid design of the $1-\mathrm{m}^{2}$ quadrat data from the Oosting Natural Area in Duke Forest, Orange County, North Carolina (Palmer and White 1994). The quadrats of the large grid are located in the lower left corner of each module of $16 \times 16 \mathrm{~m}$. Each of the three small grids contains $16 \times 16$ contiguous quadrats within a randomly selected module of the large grid. The underlying contour plot reflects relative elevation in intervals of $1 \mathrm{~m}$, ranging from $0-1 \mathrm{~m}$ (dark grey) to $10-11 \mathrm{~m}$ (white). The contour plot is derived from elevation measurements at the corner points of each module.

$$
\lambda_{f}(h)=\mathbf{u}_{f}^{\top} \mathbf{C}(h) \mathbf{u}_{f}
$$

A plot of $\lambda_{f}(h)$ against distance $h$ provides a variogram of axis $f$. It reflects the spatial covariance of complementarity explained by PCA axis $f$, or the difference in species composition due to the intrinsic gradient described by $f$.

The eigenvalue $\lambda_{f}$ of PCA axis $f$ is a weighted mean of its distance-dependent components $\lambda_{f}(h)$ :

$$
\begin{aligned}
\lambda_{f} & =\mathbf{u}_{f}^{\top} \mathbf{C} \mathbf{u}_{f}=\sum_{h} \frac{n_{h}}{n} \mathbf{u}_{f}^{\top} \mathbf{C}(h) \mathbf{u}_{f} \\
& =\sum_{h} w_{h} \lambda_{f}(h) \quad f \in\{1, \ldots, s\} .
\end{aligned}
$$

\section{Examples from the Oosting Natural Area}

The approach is illustrated with previously published data from a mixed hardwood-pine forest in North Carolina (Reed et al. 1993, Palmer and White 1994, Palmer 1995, Jonsson and Moen 1998). The study area in the Oosting Natural Area of the Duke Forest, Orange County, North Carolina, contains several forest communities with gradual transitions. The entire dataset describes the presence-absence of understory vascular plant species in a nested series of quadrats within a sampling grid of 256 modules of $16 \times 16 \mathrm{~m}$. For this paper, I analyzed the $1-\mathrm{m}^{2}$ quadrats from the main grid, 
placed in the lower-left corner of each module, and from the three randomly selected modules within which every square meter was studied (Fig. 3). In addition to plant community data, I used data on relative elevation and $\mathrm{pH}$ measurements obtained at the corners of each module (Reed et al. 1993). For the small grid 3, I estimated relative elevation at the lower-left corner of each $1-\mathrm{m}^{2}$ quadrat by linear interpolation.

To explore the effects of environmental heterogeneity, I calculated a variogram of complementarity for each of the three small grids and for the large grid by averaging half the squared Euclidean distance per distance class. To make the variograms directly comparable, I restricted the analysis to the species that occurred at least five times in each of the four data sets. A 95\% confidence interval for each variogram and distance class was estimated from the distribution of the basic elements of spatial covariance (Eq. 8). For every small grid, I estimated the mean $p_{i}$ of each of the 25 species (Eq. 12) and derived the standardized variogram of complementarity (Eq. 14).

Further analysis focused on the small grid 3, using all of its 72 species variables. I tested the significance of the departure of the variogram of species richness from the variogram of complementarity by a two-sided permutation test $(\alpha=0.05)$ with 499 permutations of the original observations, permuting each species vector independently. For each permutation, the ratio of the variance of species richness to the variance of complementarity was calculated for each distance class. The permutations thus provided a reference distribution for the ratio of each distance class under the assumption that the species are independently distributed. For the variograms of PCA axes, a variogram matrix was constructed by assembling the variograms and cross-variograms calculated from Eqs. 4 and 6 in a set of distance-dependent matrices $\mathbf{C}(h)$. The original data from the small grid 3 were subjected to PCA. Because a total of 23 axes had eigenvalues $\lambda_{f}>1$, I used a scree test (Cattell 1966) to determine the number of PCA axes to be retained. As $\lambda_{4}$ lay below the regression line fitted to $\lambda_{2}-\lambda_{23}$, the first three axes were retained for analysis. All axes were partitioned by distance class using Eq. 18. A variogram of complementarity, accounting for the variance along PCA axis 1, was obtained by summing the variograms of all other PCA axes by distance class.

All calculations were performed in S-Plus (Becker et al. 1988).

\section{RESULTS}

\section{The effect of environmental heterogeneity on spatial covariance}

How strongly does local environmental heterogeneity influence spatial covariance? The Oosting data provide an excellent example, as the four grids were sampled from a total area of only $256 \times 256 \mathrm{~m}$. The study area contained some environmental heterogeneity (cf. Fig. 3); e.g., the mean relative elevation of the four corner points of the small grids 1,2, and 3 was $3.7 \mathrm{~m}, 8.9 \mathrm{~m}$, and $7.1 \mathrm{~m}$, and their respective average pH was $6.5,5.7$, and 5.9.

Fig. 4 shows the empirical variograms of complementarity for each of the four grids, including only the 25 species that occurred at least five times in each grid. Although the four data subsets contained exactly the same species and originated from the same small study area, their variograms of complementarity differed strongly (Fig. 4). The variogram for the large grid showed a continuous rise without reaching a sill, indicating larger scale heterogeneity. The curves for the three small grids showed more or less parallel curves but approached very different sills.

The expected variance estimated from the species means in each grid predicted the different sills fairly well. This means that between-grid heterogeneity is responsible for the observed difference in the sills. The standardized variograms of complementarity in Fig. 5 (note the different scaling of the $x$ axis as compared to Fig. 4) support this interpretation, as the curves for the three grids coalesced after accounting for variable species means.

\section{Distance dependence of the variance of species richness}

Does the spatial version of the variance test of species richness provide more or better information than the global test? In the example of the small grid 3, the global variance of species richness was only slightly lower than expected under the hypothesis of independent observations and independently distributed species. Based on the global variance test, the difference was clearly not statistically significant (chi-square $=$ 248.76 , df $=256, P=0.384)$. The plot against distance, however, revealed a systematic change from predominantly negative covariances between species at small distances to mainly positive covariances at larger distances (Fig. 6). The permutation test showed significant departures of the two curves for the smallest and the largest distance classes. Hence the spatial variance test of species richness was able to detect a variance deficit at small distances undetected by the global test.

\section{Spatial structure of intrinsic gradients}

Indirect ordination methods such as PCA describe vegetation as the sum of overlapping, statistically uncorrelated intrinsic gradients. What does multiscale ordination tell us about the spatial structure of these gradients? Fig. 7 shows the variograms of PCA axes 1-3 for the small grid 3 . The variance along the first axis increased strongly and continually with distance, indicating the presence of larger scale trend. A strong correlation between axis scores and the interpolated relative elevation (Pearson correlation, $r=0.73, P<$ 


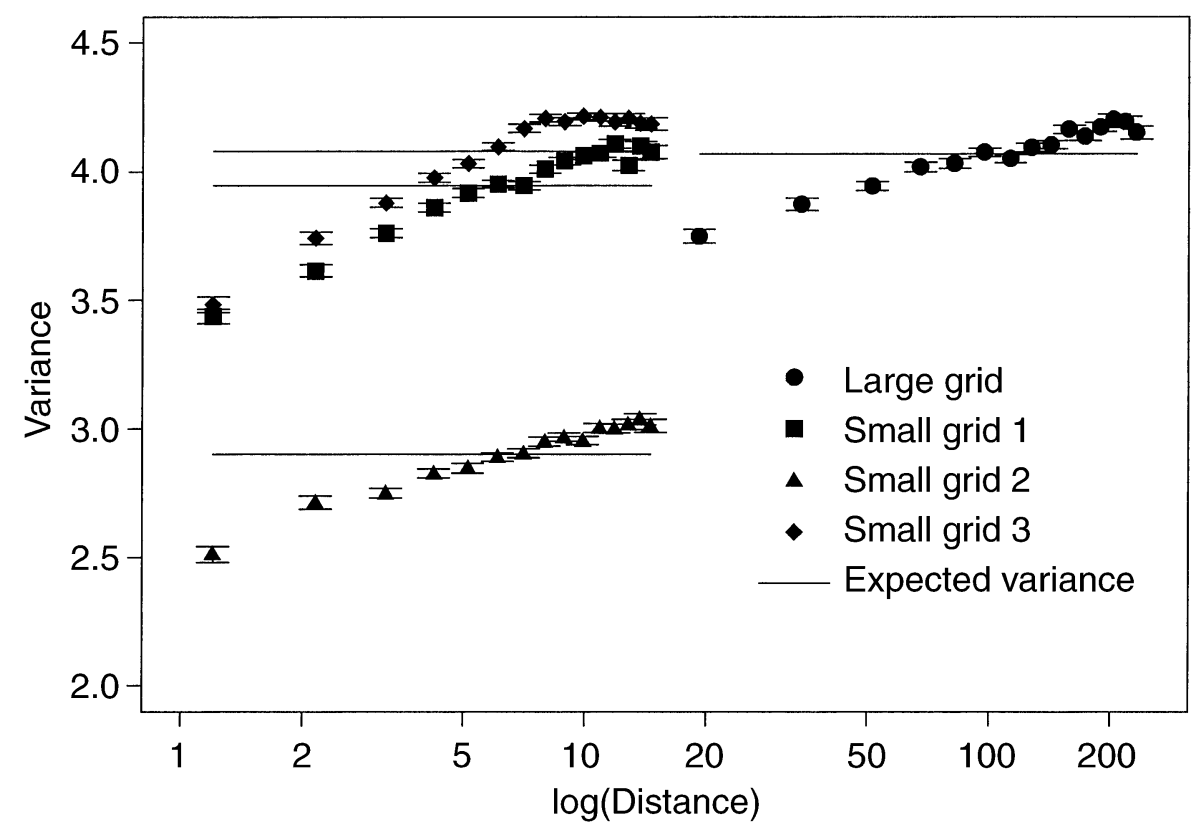

FIG. 4. Empirical variograms of complementarity for the large grid and for each of the three small grids, calculated independently for each grid. The point symbols represent the average observed semivariance per distance class, based on the 25 plant species that were present in at least five cells of each of the four grids. Error bars mark the $95 \%$ confidence interval for the mean of each distance class. The solid lines indicate the expected variance based on the species means within each grid.

0.001) suggests that the variance along PCA axis 1 is largely due to environmental heterogeneity. All other factors showed only a modest increase with distance (e.g., axis 2), or even suggested slightly cyclic patterns (e.g., axis 3) as one would expect to result from community-level processes.

How important are the factors contributing to the variance of species richness? Fig. 8 illustrates for the small grid 3 how multi-scale ordination can be used to partition the variance in a dataset into distance-dependent components of interspecific interactions, larger scale trend as caused by environmental heterogeneity, and single-species aggregation patterns. The strong increase of the variance of species richness with distance was largely due to interspecific interactions and the trend reflected in PCA axis 1. Only a small portion of the remaining variance could be explained by singlespecies aggregation patterns, peaking at $\sim 7 \mathrm{~m}$. The observed variance of complementarity without axis 1 appeared to oscillate around its global variance, indicating that all larger scale trend had been accounted for by removing PCA axis 1 .

\section{DISCUSSION}

\section{Spatial analysis of plant communities in a heterogeneous environment}

Can variograms from different communities be compared? The Oosting example illustrates the drastic effect that within-site environmental heterogeneity can have on spatial analysis. Researchers often assume ho- mogeneity over relatively small study areas. However, heterogeneity may occur at all scales, leading to differences in the mean and variance of species variables across space. The empirical variogram of the large grid did not reach a sill, indicating a violation of the secondorder stationarity assumption (e.g., Bellehumeur and Legendre 1998). The three intensively sampled modules of $16 \times 16 \mathrm{~m}$, separated by $150-250 \mathrm{~m}$, differed in their species composition and in the frequency of occurrence of the more abundant and ubiquitous species. Even when the analysis was restricted to the latter group, the resulting variograms of complementarity differed strongly in their sills due to the differences in species means between the grids.

The spatial description by a generalized variogram or "dissimilogram" (e.g., Mistral et al. 2000) would normally stop here. The mathematical approach developed here, however, allowed the different sills to be predicted from the observed species means so that the variograms could be made comparable by standardization. The standardized variograms of the three small grids were relatively well behaved, although all appeared to reach sills slightly $>1$, indicating some unaccounted internal heterogeneity. In the standardized form, the three curves coalesced and thus provided a general description of small-scale autocorrelation in species composition within the study area, independent of environmental conditions.

The variogram matrix provides two ways for dealing with larger scale heterogeneity. Eq. 11 can be used to 
FIG. 5. Standardized variograms of complementarity (cf. Eq. 14), derived independently for each of the small grids. Each point represents, for a distance class, the average of the observed squared Euclidean distance between observations divided by the expected variance based on the mean of each species in the grid. The expected sill of a standardized variogram is 1 .

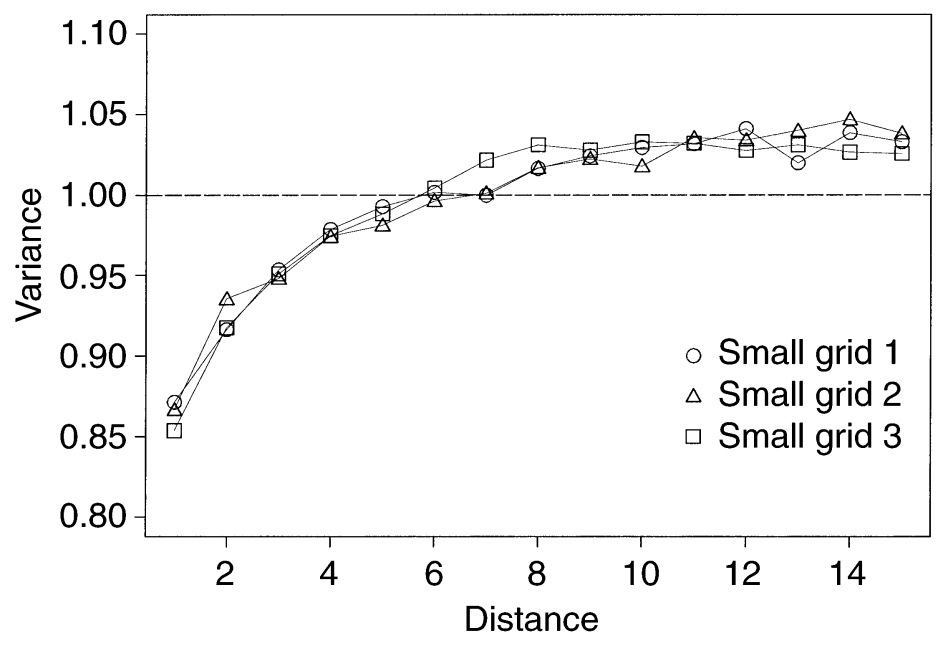

predict and account for the expected spatial covariance based on any given trend model, which may be obtained by local interpolation or by modeling species response to known environmental gradients. Multiscale ordination provides a direct way of separating variance attributed to a "true gradient" from the variance of "false gradients," as determined by the variograms of ordination axes. This approach needs to be extended to direct gradient analysis with Redundancy Analysis (RDA; Rao 1964, Legendre and Legendre 1998), where PCA axes are linear combinations of observed environmental variables.

On purely empirical grounds, however, it is not possible to distinguish between trend, or systematic variation of the mean, and autocorrelation, or local dependence of the departure from the mean. The appearance of any spatial pattern depends highly on the grain and extent of a study. The same spatial structure may appear as trend in a fine-scale study and as pattern of a specific scale if a broader extent is considered.

\section{Advantages of a spatial variance test of species richness}

The example in Fig. 5 clearly illustrates the distancedependent nature of interspecific interactions in a community and their effect on the variance of species richness. A global, nonspatial variance test is prone to miss the systematic departure of the variance of species richness from its expected value if negative covariances at small distances are cancelled out by positive covariances at larger distances. The different results of the two tests do not merely reflect their statistical power, i.e., their ability to detect a given effect with a sample of a certain size. Here, the effect itself, the global sum of the associations, increased with increasing distance between observations. In such a situation, the chances of detecting a variance deficit with the global test are likely to decrease with increasing sample size, as larger distances are included.

The negative covariance at short distances may indicate niche limitation, but it may also be an effect of
FIG. 6. Variograms of complementarity and of species richness for the small grid 3, based on all 72 species observed in that grid. Significance of the departure of the variogram of species richness from the variogram of complementarity was determined by a two-sided permutation test (see Model structure and methods: Examples from the Oosting Natural Area). The observed global variance (solid line) is the nonspatial variance of quadrat species richness, and the expected global variance (dashed line) is the sum of the (nonspatial) species variances, as used in the global version of the variance test of species richness.

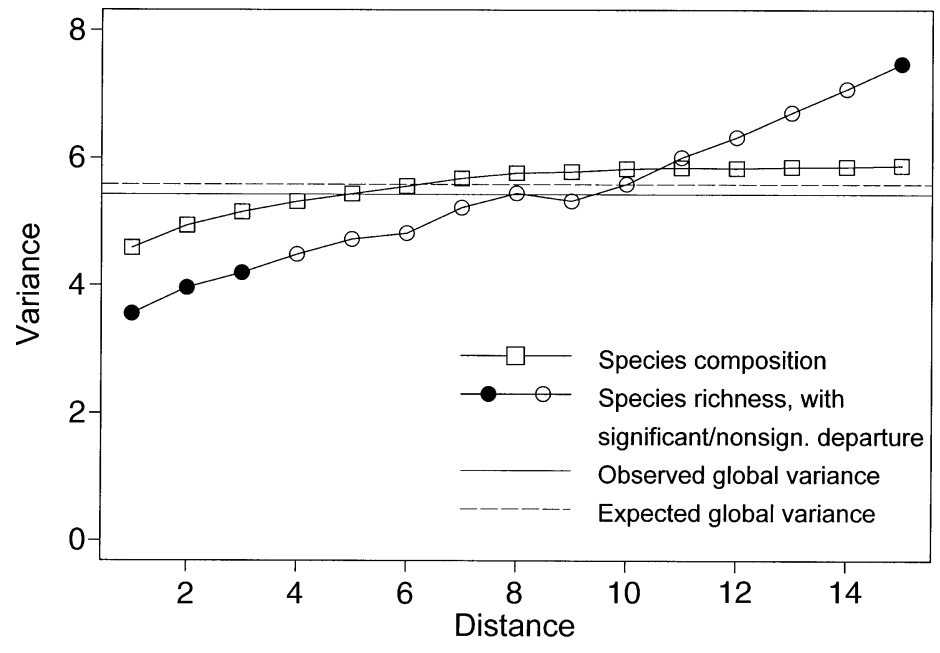




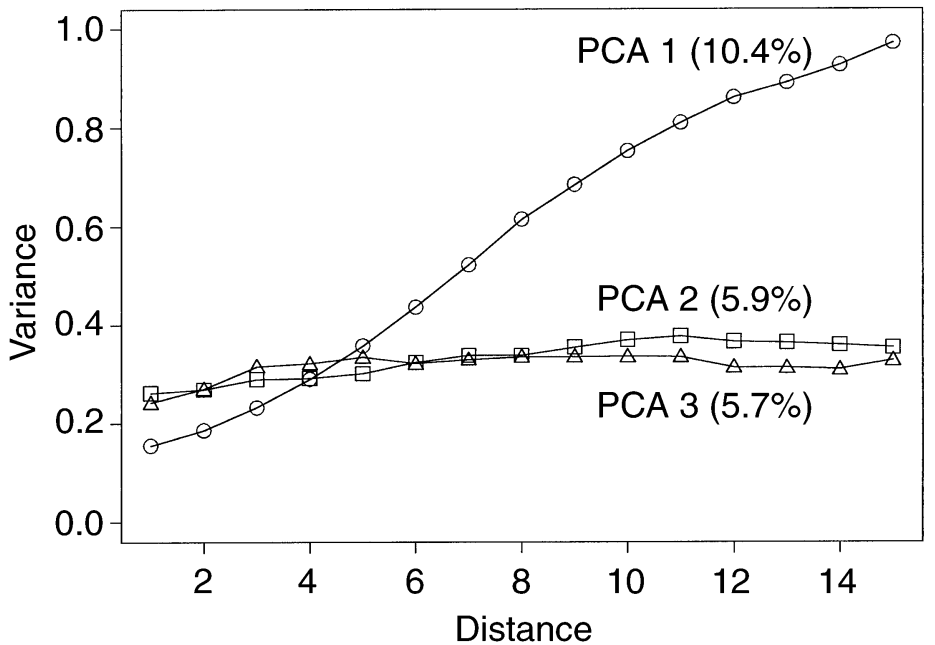

FIG. 7. Variograms of PCA axes for the small grid 3 . The lines connect the point estimates of the variance along the first, second, and third axis, respectively, as partitioned by distance using Eq. 18. The percentage of the total variance explained by the first three axes is indicated in parentheses. cell size, or of rarefaction (Økland 1994), the physical limitation of the number of individual plants and thus the number of species within relatively small areas. The increase in positive covariance with distance is probably due to increasing environmental heterogeneity, which is one of the factors determining niche limitation.

The variogram of complementarity provides a null model that already includes the autocorrelation due to single-species aggregation patterns. Hence, it eliminates the need for keeping the spatial pattern of each species constant while permuting observations. While this statement is based on mathematical considerations, it remains to be verified by a thorough empirical comparison to published permutation tests (Palmer and Van der Maarel 1995, Roxburgh and Matsuki 1999).

The spatial variance test of species richness eliminates two former impediments of observational studies of the variance deficit in species richness, namely the confounding of negative associations at short distances with positive interactions at larger distances, and the spatial autocorrelation in the distributions of the in-

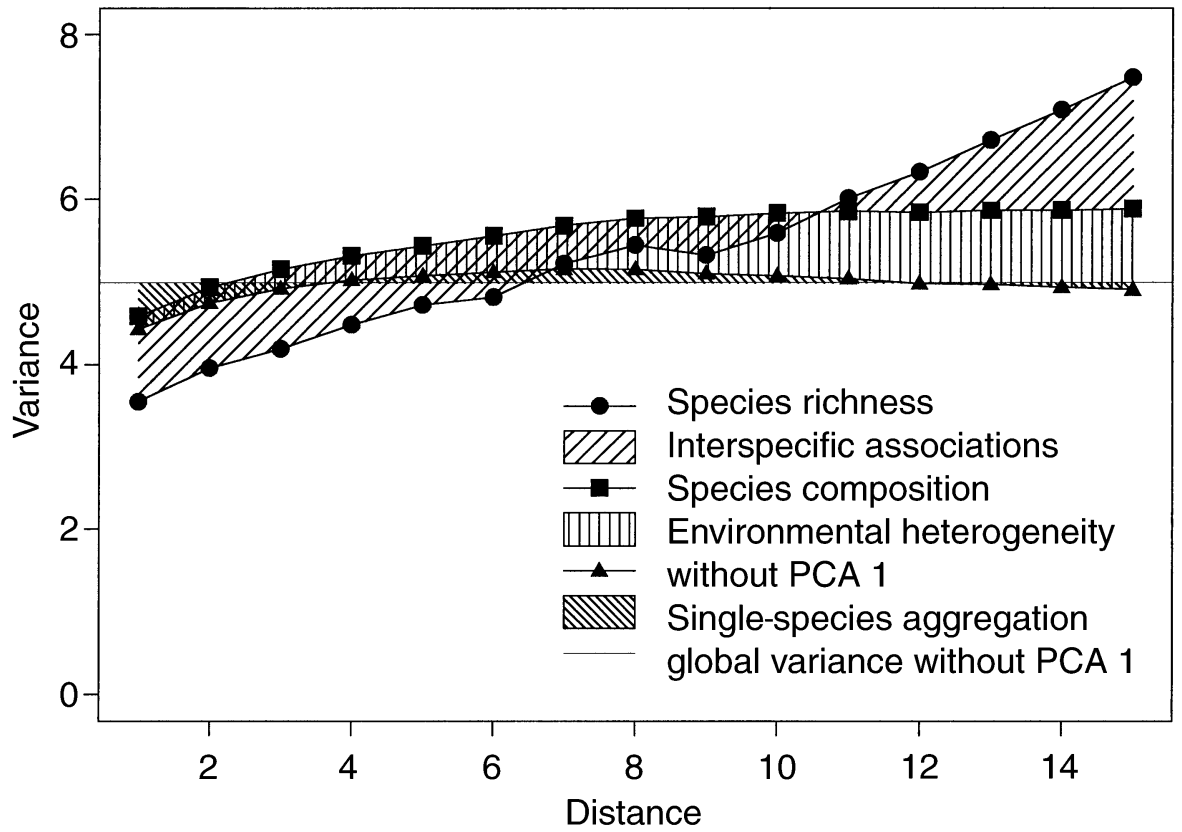

FIG. 8. Partitioning of the variance of species richness of the small grid 3 into the additive contributions of interspecific interactions, environmental heterogeneity as reflected in PCA axis 1, and single-species aggregation patterns. The point symbols represent, for each distance class, the variance of species richness, complementarity, and the variance of complementarity after accounting for PCA axis 1 as described in the text. 
dividual species. Hence, it opens the door for a substantially new approach to the research on niche limitation.

\section{A geostatistical perspective on multiscale ordination}

This paper proposes two important deviations from earlier presentations of multiscale ordination. First, Noy-Meir and Anderson (1971) suggested a simple addition of the variance matrices for each block size to obtain a global matrix that is subjected to PCA. Dale (1999) recommended weighting by the expected intensity, or amplitude of a cyclic variance, as a function of block size. Here, I suggest weighting the distance-dependent variance-covariance matrices by the number of pairs of observations in each distance class. This recreates the global, nonspatial variance-covariance matrix, which is used not only in PCA but in many multivariate methods (see worked example in the Appendix). This compatibility may lead to further integration of geostatistical modeling with nonspatial multivariate methods.

Second, in a geostatistical framework, the block size of the original definition of multiscale ordination corresponds to distance. While block sizes are defined by the analytic method, distances are a property of the sampling design and may take any value. This is a great advantage as it extends multiscale ordination to nonsystematic spatial samples, although the sampling design will still determine whether a specific pattern in an ecological community is detected by the method.

The often arbitrary choice of distance classes may, however, affect the shape of a variogram. The geostatistical solution is to analyze the variogram cloud instead of the empirical variogram defined by distance classes. The variogram cloud is a scatter plot where each dot represents the semivariance of a single pair of observations. Such a plot can easily be constructed by plotting the basic elements of spatial covariance (Eq. 8).

From a geostatistical perspective, there is another important extension. Spatial autocorrelation is often anisotropic, that is, it depends on the geographic direction in which it is measured. If the sampling design is two dimensional and the sample is large enough, directional variance-covariance matrices $\mathbf{C}(h, d)$ can be calculated, e.g., for four directional sectors $d$. However, I would expect that most cases of anisotropy in ecological data sets are due to environmental heterogeneity, so that a removal of larger scale trend would usually eliminate the need for anisotropic models.

\section{Assumptions and limitations}

Many readers may feel uncomfortable about the heavy reliance on the Euclidean distance. A main concern is that the Euclidean distance does not reflect the special nature of the value zero in biotic data. Although the Euclidean distance is especially unsuitable for cover or abundance data that include zeros (Legendre and Legendre 1998), it is more robust for presence-absence data, as illustrated in the following example: Assume that the respective abundance of species $i$ in three quadrats $a, b$, and $c$ is 4,1 , and 0 . Based on the squared Euclidean distance, $D^{2}, b$ and $c$ are more similar $\left(D^{2}\right.$ $=1)$ than are $a$ and $b\left(D^{2}=9\right)$, although $a$ and $b$ share species $i$, which is absent from $c$ (Legendre and Legendre 1998). However, converted to presence-absence data, $b$ and $c$ are more different $\left(D^{2}=1\right)$ than $a$ and $b\left(D^{2}=0\right)$.

A zero may be stochastic (the sampling unit represents suitable habitat, but is unoccupied) or it may be structural (unsuitable habitat, where the species cannot occur). Structural zeros, which can inflate observed species associations, occur if the sample includes a large degree of environmental heterogeneity. In such a situation, local means should be accounted for in the modeling of variograms (Eq. 11). If a multiscale ordination is performed, this adjustment may not be enough. PCA assumes that the individual species are linearly related to the main gradients in species composition. If there is a considerable degree of beta diversity, as a result of environmental heterogeneity, species may appear and disappear along a compositional gradient. In order to accommodate such a unimodal response or optimum curve, the variogram matrix approach needs to be generalized and extended to a chisquare measure of distance that is compatible with correspondence analysis (CA; cf. Legendre and Legendre [1998] for an overview of chi-square based ordination methods). An approach based on the chi-square distance would also accommodate abundance data.

The Euclidean distance has some distinct advantages. First, it leads to a direct mathematical extension of the variogram and is compatible with multivariate geostatistical methods used in other fields for quantitative variables (e.g., Goovaerts [1994] in soil science, Myers [1997] in remote sensing). Second, it mathematically links the spatial structure of individual species variables, of complementarity, and of species richness. A similar partitioning may be possible for other resemblance measures, such as the chi-square distance. The direct ecological interpretations, however, and specifically the explicit decomposition of the variance of species richness, are unique to the Euclidean distance.

The biggest advantage of the Euclidean distance is its general (though often implicit) use in statistics, which makes the variogram matrix approach highly generalizable. Any variable that is the sum of other variables can be defined and interpreted as the sum of a variance-covariance matrix. If variance is calculated from pairwise differences instead of individual differences from the mean, any variance or variance-covariance matrix can be partitioned by distance.

A last concern is that the observed spatial covariance will strongly depend on quadrat size, as Palmer and White (1994) illustrated with additional data collected at eight nested quadrat sizes from the Oosting study area. Scale dependence has many faces and aspects, 
which can be related to the two basic components of scale, grain, and extent (Wiens 1989). While most block-size variance techniques manipulate grain, or quadrat size, geostatistical analysis focuses on extent, or distance between observations. A parallel geostatistical analysis at different cell sizes may help to untangle the effects of grain and extent on ecological patterns and processes.

\section{Conclusions}

Variance in ecological communities and systems is spatially structured. This is true for abiotic and biotic factors, for populations, communities, and biodiversity. The ecological literature most often treats spatial structure as a problem rather than as information, stressing problems for significance tests due to spatial autocorrelation instead of the additional insights that can be gained from spatial analysis (cf. Legendre 1993). When ecologists analyze variation in plant communities, they either focus on plant-environment interactions using ordination methods that ignore spatial structure, or they describe the pattern in one or two species without taking into account the spatial structure caused by environmental factors or community-level processes.

The composition and diversity of a plant community results from processes at different levels of biological organization operating in a spatially structured environment. As the Oosting example illustrated, the patterns created by abiotic and biotic processes overlap to form a complex spatial covariance structure that is difficult to compare or interpret. However, the joint analysis of single-species, interspecific, and abiotic effects revealed component patterns that were highly interpretable. This shows that an integrated methodological approach is needed to understand what determines community structure, and ultimately, why species coexist.

The variogram matrix is a geostatistical extension of multiscale ordination. It partitions the variance in ecological communities into spatial components on the levels of individual populations, species composition, and species richness, and can be used to factor out the effects of single-species aggregation patterns, interspecific interactions, or environmental heterogeneity. By integrating three traditionally unrelated methods, it increases the interpretability of variograms of plant communities, provides a spatial extension and an empirical null model for the variance test of species richness, and extends multiscale ordination to nonsystematic spatial samples.

Beyond the individual applications, the variogram matrix provides a framework for the mathematical unification of geostatistics, multivariate data analysis, and the analysis of variance. An integration of geostatistics with general multivariate statistical methods may enable ecologists from a broad range of fields to incorporate spatial structure and processes into their re- search and to integrate analyses across different levels of biological organization.

\section{ACKNOWLEDGMENTS}

This research is part of a project funded by the Environmental Protection Agency (EPA) under STAR grant No. R826764-01-0. Additional funding came from the Swiss National Science Foundation (SNF), NCCR Plant Survival, grant No. 5005-65748. Mike Palmer, Oklahoma State University, provided the Oosting data sets and many valuable comments. Robert Peet, University of North Carolina at Chapel Hill, provided the Oosting elevation data. Jennifer Hoeting, John Wiens, Bea Van Horne, and Jonathan Bossenbroek, all at Colorado State University, P. A. Burrough at University of Utrecht, and Sucharita Ghosh at WSL Swiss Federal Research Institute, provided important critique and support. I would like to thank David Roberts, Bengt Gunnar Jonsson, and an anonymous reviewer for their valuable comments.

\section{LiterATURE Cited}

Allen, T. F. H., AND T. B. Starr. 1982. Hierarchy-perspectives for ecological complexity. University of Chicago Press, Chicago, Illinois, USA.

Becker, R. A., J. M. Chambers, and A. R. Wilks. 1988. The NEW S Language. Chapman and Hall, New York, New York, USA.

Bellehumeur, C., and P. Legendre. 1998. Multiscale sources of variation in ecological variables: modeling spatial dispersion, elaborating sampling designs. Landscape Ecology 13:15-25.

Burrough, P. A. 1987. Spatial aspects of ecological data. Pages 213-251 in R. H. G. Jongman, C. J. F. ter Braak, and O. F. R. van Tongeren, editors. Data analysis in community and landscape ecology. Cambridge University Press, Cambridge, UK.

Burrough, P. A., and R. A. McDonnell. 1998. Principles of geographical information systems. Oxford University Press, Oxford, UK.

Cattell, R. B. 1966. The scree test for the number of factors. Multivariate Behavioral Research 1:245-276.

Colwell, R. K., and J. A. Coddington. 1994. Estimating terrestrial biodiversity through extrapolation. Philosophical Transactions of the Royal Society of London, B, Biological Sciences 345:101-118.

Cressie, N. A. C. 1991. Statistics for spatial data. Wiley, New York, New York, USA.

Dale, M. R. T. 1999. Spatial pattern analysis in plant ecology. Cambridge University Press, Cambridge, UK.

De'ath, G. 1999. Principal curves: a new technique for indirect and direct gradient analysis. Ecology 80:2237-2253.

Fortin, M.-J. 1999. Spatial statistics in landscape ecology. Pages 253-279 in J. M. Klopatek and R. H. Gardner, editors. Landscape ecological analysis: issues and applications. Springer-Verlag, New York, New York, USA.

Galiano, E. F. 1983. Detection of multi-species patterns in plant populations. Vegetatio 53:129-138.

Goovaerts, P. 1994. Study of spatial relationships between two sets of variables using multivariate geostatistics. Geoderma 62:93-107.

Greig-Smith, P. 1952. The use of random and contiguous quadrats in the study of structure in plant communities. Annals of Botany 16:293-316.

Haining, R. 1997. Spatial data analysis in the social and environmental sciences. Cambridge University Press, Cambridge, UK.

Hill, M. O. 1973. Intensity of spatial pattern in plant communities. Journal of Ecology 61:225-232.

Isaaks, E. H., and R. M. Srivastava. 1989. An introduction to applied geostatistics. Oxford University Press, New York, New York, USA. 
Jonsson, B. G., and J. Moen. 1998. Patterns in species association in plant communities: the importance of scale. Journal of Vegetation Science 9:327-332.

Kershaw, K. A. 1964. Quantitative and dynamic ecology. Edward Arnold, London, UK.

Koenig, W. D. 1999. Spatial autocorrelation of ecological phenomena. Trends in Ecology and Evolution 14:22-26.

Legendre, P. 1993. Spatial autocorrelation: Trouble or new paradigm? Ecology 74:1659-1673.

Legendre, P., and M.-J. Fortin. 1989. Spatial pattern and ecological analysis. Vegetatio 80:107-138.

Legendre, P., and L. Legendre. 1998. Numerical ecology. Elsevier, Amsterdam, The Netherlands.

McCulloch, C. E. 1985. Variance tests for species association. Ecology 66:1676-1681.

Mistral, M., O. Buck, D. C. Meier-Behrmann, D. A. Burnett, T. E. Barnfield, A. J. Scott, B. J. Anderson, and J. B. Wilson. 2000. Direct measurement of spatial autocorrelation at the community level in four plant communities. Journal of Vegetation Science 11:911-916.

Myers, D. E. 1997. Statistical models for multiple-scaled analysis. Pages 273-293 in D. A. Quattrochi and M. F. Goodchild, editors. Scale in remote sensing and GIS. Lewis, New York, New York, USA.

Nekola, J. C., and P. S. White. 1999. The distance decay of similarity in biogeography and ecology. Journal of Biogeography 26:867-878.

Noy-Meir, I., and D. Anderson. 1971. Multiple pattern analysis or multiscale ordination: towards a vegetation hologram. Pages 207-232 in G. P. Patil, E. C. Pielou, and E. W. Water, editors. Statistical ecology: populations, ecosystems, and systems analysis. Pennsylvania State University Press, University Park, Pennsylvania, USA.

Økland, R. H. 1994. Patterns of bryophyte associations at different scales in a Norwegian boreal spruce forest. Journal of Vegetation Science 5:127-138.

Palmer, M. W. 1988. Fractal geometry: a tool for describing spatial patterns of plant communities. Vegetatio 75:91-102.

Palmer, M. W. 1995. How should one count species? Natural Areas Journal 15:124-135.

Palmer, M. W., and E. Van der Maarel. 1995. Variance in species richness, species association, and niche limitation. Oikos 73:203-213.
Palmer, M. W., and P. S. White. 1994. Scale dependence and the species-area relationship. American Naturalist 144: 717-740.

Rao, R. C. 1964. The use and interpretation of principal component analysis in applied research. Sankhyaá, Series A 26:329-358.

Reed, R. A., R. K. Peet, M. W. Palmer, and P. S. White. 1993. Scale dependence of vegetation-environment correlations: a case study of a North Carolina piedmont woodland. Journal of Vegetation Science 4:329-340.

Rossi, R. E., D. J. Mulla, A. G. Journel, and E. H. Franz. 1992. Geostatistical tools for modeling and interpreting ecological spatial dependence. Ecological Monographs 62: 277-314.

Roxburgh, S. H., and M. Matsuki. 1999. The statistical validation of null models used in spatial association analyses. Oikos 85:68-78.

Schluter, D. 1984. A variance test for detecting species associations, with some applications. Ecology 65:998-1005.

Sokal, R. R. 1986. Spatial data analysis and historical processes. Proceedings of the Fourth International Symposium of Data Analysis and Informatics, Versailles, France. North Holland, Amsterdam, The Netherlands.

Usher, M. B. 1975. Analysis of pattern in real and artificial plant populations. Journal of Ecology 63:569-586.

Van der Maarel, E., V. Noest, and M. W. Palmer. 1995. Variation in species richness on small grassland quadrats: niche structure or small-scale plant mobility? Journal of Vegetation Science 6:741-752.

Vane-Wright, R. I., C. J. Humphries, and P. H. Williams. 1991. What to protect? - Systematics and the agony of choice. Biological Conservation 55:235-254.

Ver Hoef, J. M., N. A. C. Cressie, and D. C. Glenn-Lewin. 1993. Spatial models for spatial statistics: some unification. Journal of Vegetation Science 4:441-452.

Ver Hoef, J. M., and D. C. Glenn-Lewin. 1989. Multiscale ordination: a method for detecting pattern at several scales. Vegetatio 82:59-67.

Wiens, J. A. 1989. Spatial scaling in ecology. Functional Ecology 3:385-397.

Wilson, J. B., R. K. Peet, and M. T. Sykes. 1995. What constitutes evidence of community structure? A reply to van der Maarel, Noest and Palmer. Journal of Vegetation Science 6:753-758.

\section{APPENDIX}

A worked example of spatial covariance in plant communities is available in ESA's Electronic Data Archive: Ecological Archives E084-023-A1. 